$63^{\text {ème }}$ Congrès de la SFCO, 03026 (2015)

DOI:10.1051/sfco/20156303026

(C) Owned by the authors, published by EDP Sciences, 2015

\title{
Apport de la chirurgie et de l'orthodontie dans le traitement des molaires permanentes incluses
}

\author{
Catherine J-H***, Massereau E*, Trieu J-Y*, Ordioni U*, Le Gall M* \\ * Service d'Odontologie, Assistance Publique Hôpitaux de Marseille, Hôpital de la Timone, 264 rue Saint \\ Pierre, 13005 Marseille \\ ** UMR 7268 ADES, Aix-Marseille Université/EFS/CNRS, Faculté de Médecine- secteur Nord, Bd. Pierre \\ Dramard, 13344 Marseille Cedex 15
}

L'inclusion des deuxièmes et des premières molaires est rare avec une prévalence de 0,06 à 2,3\% (Cassetta et al. 2013). Celle-ci est en augmentation depuis ces deux dernières décennies (Fu et al. 2012, Cho et al. 2008). Plusieurs étiologies peuvent être retrouvées dans ces anomalies de l'éruption, des causes générales (facteurs phylogénétiques, hérédité...) ou locales (mésio-position, taurodontisme, ankylose...) mais le plus souvent la cause de ces inclusions restent idiopathique. L'imagerie tridimensionnelle (Tyndell et Rathore 2008) a permis une meilleure compréhension de l'anatomie et de la topographie de ces dents et offre une aide au diagnostic et au traitement.

Plusieurs traitements sont décrits dans la littérature (Shpack et al. 2013, Lau et al. 2013) allant de l'abstention thérapeutique et la surveillance à la mise en place par une égression chirurgicale voir l'extraction de la dent incluse avec ou sans extraction de la dent de sagesse pour gérer l'espace disponible dans les cas d'encombrement postérieur. Des cas d'auto transplantation sont également rapportés.

Le plus souvent le traitement associera un traitement orthodontique et chirurgical. La mise en place de séparateur élastique permet parfois de lever l'obstacle et de laisser la molaire faire son éruption. Le repositionnement chirurgical permet une mise en place rapide mais présente des risques pour la vitalité pulpaire et le desmodonte de la dent incluse.

Un traitement par égression orthodontique avec le collage d'une attache sur la face vestibulaire ou occlusale reste l'alternative de choix.

Les progrès des moyens d'ancrage intra-osseux (mini-vis ou plaque) permettent désormais de traiter des inclusions profondes en évitant les pertes d'ancrage et leurs effets secondaires (ingression, rotation, résorptionradiculaire...). Le choix du traitement est discuté en fonction des critères d'âge, d'inclusion de la dent, de motivation du patient et de pronostic. La littérature reste insuffisante pour prédire le pronostic de chaque traitement (Kenrad et al. 2011). Elle rapporte le plus souvent des cas uniques. Plusieurs cas cliniques sont présentés pour discuter des indications, des avantages et des inconvénients des différents traitements.

Une collaboration étroite entre le chirurgien oral et l'orthodontiste est nécessaire pour répondre avec succès à ce challenge.

This is an Open Access article distributed under the terms of the Creative Commons Attribution License 4.0, which permits unrestricted use, distribution, and reproduction in any medium, provided the original work is properly cited. 
$63^{\text {ème }}$ Congrès de la SFCO

Nom et adresse du conférencier

Jean-Hugues CATHERINE

Assistance Publique Hôpitaux de Marseille, Hôpital de la Timone

264 rue Saint Pierre

13005 Marseille (France)

Jean-hugues.catherine@univ-amu.fr 